\title{
Soft X-Ray Tomography: Filling the Gap Between Light and Electrons for Imaging Hydrated Biological Cells
}

\author{
Lucy M. Collinson ${ }^{1}$, Marie-Charlotte Domart ${ }^{1}$, Raffaella Carzaniga ${ }^{1}$, Minoo Razi ${ }^{1}$, Peter Guttmann ${ }^{2}$, \\ Gerd Schneider $^{2}$, Eva Pereiro ${ }^{3}$, Sharon A Tooze ${ }^{1}$, Elizabeth Duke ${ }^{4}$ \\ 1. The Francis Crick Institute, 1 Midland Road, London, UK \\ 2. Helmholtz-Zentrum Berlin für Materialien und Energie GmbH, Institute for Soft Matter and Functional \\ Materials, Berlin, Germany \\ 3. ALBA Synchrotron Light Source, 08290 Cerdanyola del Valles, Barcelona, Spain \\ 4. Diamond Light Source, Harwell Science and Innovation Campus, Didcot, Oxon, UK.
}

Fluorescence microscopy is a powerful tool for localising proteins within biological samples. However, information is limited to the distribution of the tagged protein, telling us little about the ultrastructure of the surrounding cells and tissues, which may be intimately involved in the biological process under study.

Electron microscopy (EM) overcomes the resolution limitation inherent in light microscopy and can reveal the ultrastructure of cells and tissues. EM can be performed at high resolution and across significant volumes, using a range of systems, from transmission EM (TEM) to serial block face scanning EM (SBF SEM) and focused ion beam scanning EM (FIB SEM) [1]. However, chemically-fixed and resin-embedded samples suffer from a range of artifacts, particularly related to the dehydration process, such as shrinkage and extraction of molecules. To avoid dehydration, samples can be vitrified by plunge freezing and high pressure freezing, which preserves cells and tissues in their fully hydrated state. These vitrified samples can be imaged in TEMs and FIB SEMs equipped with cooled stages and using low dose imaging regimes. Whilst ultrastructure is improved, cryo-techniques come with their own set of technical challenges. Contamination-free transfer between cryo-preparation and cryo-imaging systems is non-trivial, as is imaging samples thicker than $\sim 1 \mu \mathrm{m}$.

Cryo soft X-ray tomography (cryo SXT) complements light and electron microcopy, filling the niche of near-native state ultrastructural imaging of vitrified cells and tissues through depths of 1-10 $\mu \mathrm{m}[2,3,4,5]$. Experiments were performed at the soft X-ray transmission microscopes installed at the U41 beamline at the BESSYII electron storage ring (Berlin, Germany), the MISTRAL beamline at the ALBA synchrotron (Barcelona, Spain) and the B24 beamline at the Diamond Light Source (Harwell, UK). Cryo SXT allows us to capture the most volatile cellular structures, perform accurate volumetric measurements at the nanoscale, follow organelle connectivity over many microns, and perform elemental analyses of cellular compartments .

Ultrastructural analysis of cells and tissues using cryo SXT can be extended with functional information by correlating with cryo-fluorescence imaging of fluorescently-tagged molecules expressed in cells. I will illustrate the application of this powerful correlative technique with a study of autophagy, a critical cellular process in cell health and disease [6,7]. In the future, these studies would benefit from the development of super-resolution cryo-fluorescence for high resolution correlation, and the addition of cryo-TEM of targeted regions to add macromolecular detail to whole cell ultrastructure. In the near future, we are likely to see the arrival of lab-based cryo SXT microscopes that could increase throughput in sample screening and data collection, to target key samples for precious synchrotron experiments. 


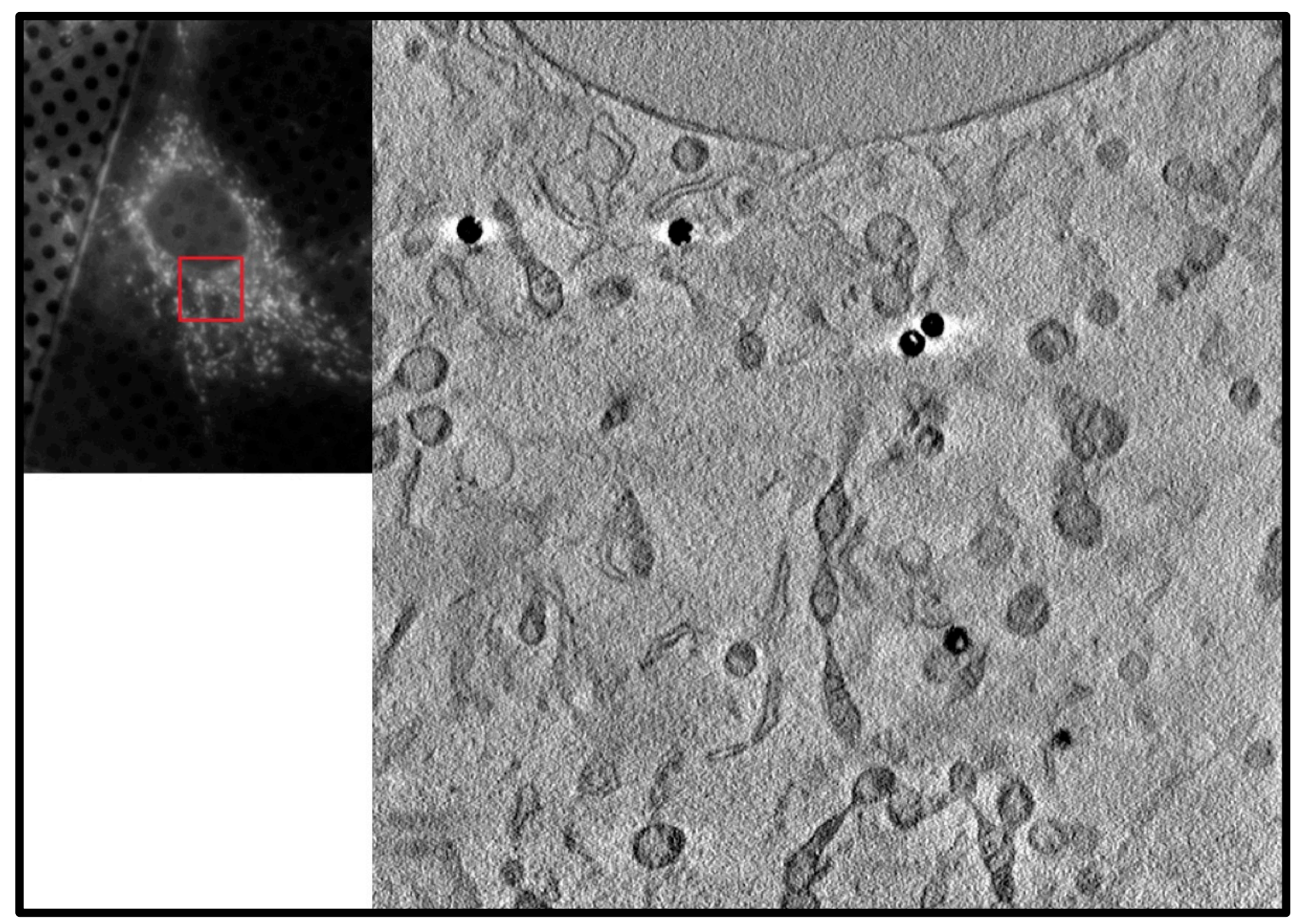

Figure 1. A whole vitrified HeLa cell, imaged using a correlative cryo-fluorescence (left) and cryo-soft Xray tomography (right) workflow at the MISTRAL beamline at the ALBA synchrotron.

\section{References}

[1] Peddie, C.J. \& Collinson, L.M. (2014) Micron. 61,9-19.

[2] Schneider, G. et al. (2010) Nat.Methods.12,985-7.

[3] Carzaniga, R. et al. (2013) Protoplasma.251,449-58.

[4] Carzaniga, R. et al. (2014) Methods Cell Biol.124,151-78.

[5] Duke, E. et al. (2014) J.Microscopy.255,65-70.

[6] Duke, E. et al. (2014) Ultramicroscopy.143,77-87.

[7] Orsi, A., et al. (2012) MBoC.23:1860-73

[8] This work was supported by the Francis Crick Institute, which receives its core funding principally from Cancer Research UK (FC001999), the UK Medical Research Council (FC001999) and the Wellcome Trust (FC001999). The research leading to these results has received funding from the European Community's Seventh Framework Programme (FP7/2007-2013) under BioStruct-X (grant agreement $\mathrm{N}^{\circ} 283570$ ). 